\title{
The Impact of Computational Pharmacology
}

\author{
Matthew N.O. Sadiku, PhD \\ Sheena M. Reeves, PhD \\ Sarhan M. Musa, PhD \\ Roy G. Perry College of Engineering, \\ Prairie View A\&M University, Prairie View, TX, USA
}

Doi: 10.19044/esj.2019.v15n9p151 URL:http://dx.doi.org/10.19044/esj.2019.v15n9p151

\begin{abstract}
Pharmacology is the study of how drugs interact within the human body. The field covers a variety of topics such as pharmaceutical capabilities and interactions. Early studies in pharmacology focused on the effects of natural substances in the body as a means of therapeutic treatment. Modern pharmacology uses computation and modeling as research tools on a cellular level. Computational models are useful in almost every scientific and engineering discipline especially when practical and ethical considerations prevent experimenting with real systems. The models are needed to design various parts of the drug discovery process. Computer programs for designing compounds is one key area of computational pharmacology. Another area of interest are digital repositories for investigating chemical interactions. Modern pharmacology now involves using a computational method. This paper provides a brief introduction to computational pharmacology.
\end{abstract}

Keywords: Computational pharmacology, computational drug discovery, computer-aided drug design

\section{Introduction}

As the number of people in the world increases, the number of people seeking medical attention for a variety of reasons also increases. The higher frequency of diseased individuals has resulted in hospitals, clinics, and other medical-related establishments, such as pharmaceutical companies, becoming inundated with filed data sets which may include electronic health records of patients and clinical reports from drug trials (Bellai, 2011). Rare diseases are also more prominent in recent years as seen in the outbreak of the polio-like disease, acute flaccid myelitis or AFM, which has paralyzed hundreds of American children in the last five years (McKay, 2018). According to the Centers for Disease Control and Prevention (CDC), several factors and 
symptoms have been found in patients; however, all are not found in all patients (McKay, 2018). It was believed that the enterovirus D68 or EV-D68 was the main cause of AFM but results have been inconclusive as all cases do not possess this result (Pastula, 2014; Nelson, 2016; Morrey, 2018). There are many questions that remain about the disease such as is source and possible treatment mechanisms.

Pharmacology is the study of the interaction between drugs and biological systems. This area of science includes topics such as medicine, pharmacy, nursing, and dentistry. Two main focus areas are pharmacodynamics and pharmacokinetics (Lees, 2004). Pharmacodynamics is defined as the study of the molecular, biochemical, and physiological effects of drugs. Pharmacokinetics is the study of how drugs move through the body whether it is the absorption process, drug distribution, and excretion of drugs (Lees, 2004).

Pharmacology can be further subdivided neuropharmacology, cardiovascular pharmacology, molecular pharmacology, biochemical pharmacology, clinical pharmacology, psychopharmacology, and chemotherapy (Weinhart, 2018). Some areas focus on a different part of the human body such as the heart, blood vessels, or nervous system. Other areas are more concerned with patient treatment such as clinical pharmacology or patient psyche such as psychopharmacology.

Modern pharmacology seeks the usage of computation and modeling as research and design tools. Computational and simulation approaches provide modeling tools to study biological systems and the effect that drugs have on them (Weinhart, 2018). The following text reviews the rapidly growing number of fields switching to the usage of computational methods and the areas of application.

\section{Computation as a necessity}

Over the years, the advances and success rate in the production of new drugs has dwindled in the face of new medical challenges (Cressey, 2011; Pammolli, 2011). Most pharmaceutical companies will spend over \$1 billion in hopes of introducing a new drug to the market (Kaitin, 2010). Due to the economic considerations in the development of new drugs, there are concerns regarding the suitability of maintaining current approaches in pharmacological research (Weinhart, 2018). In short, the experimental methods are expensive. Classic medical treatments research focused solely on developing a drug for a single purpose or target. Theoretical pharmacologists aim at finding the structure - activity relations of a particular drug and predicting the biological activity of the drug. Recently, computational analysis emerged as a useful approach for drug design and discovery as a predictive model (Vilar, 2014; Lui, 2014; Hodos, 2016; Platamia, 2018). 
Computational calculations supplement theoretical physics and experimental physics. Since most physics problems are difficult to solve exactly or may be nonlinear in nature, design is required. Today computation is considered as an equal partner with theory and experiment.

Computation provides a means of studying complex natural phenomena that would be too expensive or dangerous to study by direct experimentation. As the capabilities of computers increase, computational methods and analysis have become crucial in the simulation of physical events and engineering systems. Computational experimentation involves computer simulation and is often regarded as computer experimentation or simply computer modeling. Computational models are useful as these models are often implemented in common programming language such as $\mathrm{C}++$, Basic, or FORTRAN. Development of such models can occur using software packages such as MATLAB or MATHEMATICA.

Many disciplines have undergone a computational methods overhaul in the past decades. The recent computational turn has produced several new fields of studies including: computational biology, computational chemistry, computational mechanics, computational fluid dynamics, computational physics, computational electromagnetic, computational humanities, and computational finance (Sadiku, 2018). Computation also plays a vital role in the pharmacology industry.

Computational pharmacology (CP) involves the integrated study of pharmacology and computational analysis. This field involves computational approaches for the storage of pharmacology analysis and sensitive information, management of drug trials, analysis of results, and modeling of drug systems. CP allows for probing the mechanisms of drug interactions that often occurs in one of the excitable diseases. CP is commonly used as a predictive tool for large data sets as seen in modeling drug-to-drug interactions (Vilar, 2014). CP has the capability of providing a 'multi-angled' approach to predicting drug interactions in the body (Hodos, 2016).

Review of the literature suggests an apparent 'gap' between experimental and computational methods (Babcok, 2013). Human-based models are designed to bridge the gap from basic research to preclinical drug testing (Weinhart, 2018). Computational models provide several economic advantages for drug development by refining drug design (Lui, 2014). The following section reviews some recent findings in CP applications based on experimental work.

\section{CP applications}

Computational tools are routinely developed to address drug-related problems. These tools efficiently assess potential new interactions and predict unknown drug-target interaction. One source groups $\mathrm{CP}$ applications into three 
main categories (Hodos, 2016): 1) prediction of drug-to-target interactions, 2) prediction and characterization of drug side effects, and 3) repurposing of drugs. One study involved a systemic computational pharmacology approach using pharmacokinetic modeling that was introduced to investigate the global nature of certain drugs such as Traditional Chinese medicine (TCM) (Wu, 2011). This study proved that CP enhanced understanding of a complex system. Another study for CP utilized the technology in drug repurposing. Hodos et. al (2016) hypothesized that CP matrix factorization is an acceptable approach in integrating diverse data and determining missing data.

Drug interaction is often complex and can have a wide range of parameters that affect the overall performance of the drug (Boran, 2010; Vilar, 2014; Hobos, 2016). Additional models have been developed that address other drug properties and design parameters such as rate of absorption, bioavailability, binding tendencies, metabolism, volume of distribution, and other drug interactions. Common methods of developing these statistical models include data regression or neural networks (Sliwoski, 2014).

The next step in evolving CP capabilities is understanding how to successfully integrate multiple data sets in a manner that provides understanding of human disease (Boran, 2010). One area of interest includes performing qualitative and quantitative image analysis of diseases cells. Another area includes the analysis and modeling of large genomic and proteomic data sets. $\mathrm{CP}$ is also useful in designing novel molecular tools to probe and perturb cellular function and developing predictive models of complex cellular processes.

The future of computational pharmacology also includes the development of personalized medicine applications based on algorithms configured for a single individual (Hodos, 2016). This type of technology can advance treatment for individuals with diseases that can vary by patient such as cancer, sickle cell anemia, or diabetes. For example, diabetic retinopathy is a diabetic disease that currently possesses no approved early stage drug treatments (Platania, 2018). A recent study by Platiania et. al (2018) has utilized a biological based CP to analyze the diabetic retinas in some animals. The group deemed the process as a useful biological CP tool and suggested that the method was suitable for other pathological investigations.

\section{Conclusion and outlook}

Computational approaches in pharmacology offer a means of identifying potential interactions. Computational pharmacology has been proven as a useful and effective technique for finding, mining, and predicting the properties of Chinese medicine. The method is involved in leading current medical advances. Predictive models using multiple parameters have been proven successful as the case with Vilar et. al (2014). 
In spite of the progress in computational pharmacology, several challenges remain unsolved. Several diseases, such as cancer, autism, and depression, remain without a cure and are, sometimes, not adequately treated. The pharmaceutical industry and their regulating bodies have recognized the importance of computational pharmacology in today's society. More occurrences of rare diseases, such as the recent acute flaccid myelitis (AFM) outbreak in U.S. children, has led to the increasing need for more expertise in CP. It is imperative that more universities seek to train graduates will use their knowledge of CP to bridge knowledge gaps as members of multidisciplinary research groups. The advancement of computation pharmacology is without a doubt important to future pharmaceutical discoveries.

\section{References:}

1. Babcock, J.J. (2013). Computational pharmacology of the HERG potassium channel, Doctoral Dissertation, Johns Hopkins University.

2. Bellazzi, R., Diomidous, M., Sarkar, I.N., Takabayashi, K., Ziegler A., and A.T. McCray (2011). Data analysis and data mining: current issues in biomedical informatics. Meth. Info. Med. 50, p. 536.

3. Boran, A.D.W. and R. Iyengar (2010). Systems approaches to polypharmacology and drug discovery, Curr. Op. Drug Discov. Dev., 13(3), pp. 297-309.

4. Cressey, D. (2011). Traditional drug-discovery model ripe for reform. Nature, 471, pp. 17-18.

5. Hodos, R.A., Kidd, B.A., Khader, S., Readhead, B.P., and J.T. Dudley (2016). Computational Approaches to Drug Repurposing and Phamacology, Wiley Interdiscip. Rev. Syst. Biol. Med. 8(3), pp. 186210.

6. Kaitin, K.I., (2010). Deconstructing the drug development process: the new face of innovation. Clin. Pharmacol. Thera., 87, pp. 356-361.

7. Lees, P., Cunningham, F.M., and J. Elliott (2004). Principles of pharmacodynamics and their applications in veterinary pharmacology. J. of Veter. Pharmacol. and Thera., 27(6), pp. 397-414.

8. Liu, C. (2014). Computational approaches in molecular and systems pharmacology: Application to neurosignaling membrane proteins, Master's Thesis, University of Pittsburgh.

9. McKay, S.L., Lee, A.D., Lopez, A.S., Nix, W.A., Doolin, K.L., Keaton, A.A., Spence-Davizon, E., Herlihy, R., Clark, T.A., Hopkins, S.E., Pastula, D.M., Sejvar, J., Oberste, M.S., Pallansch, M.A., Patel, M., and J.A. Routh (2018). Increase in Acute Flaccid Myelitis United States, 2018. MMWR Morb Mortal Wkly Rep. 67, pp. 12731275. DOI: http://dx.doi.org/10.15585/mmwr.mm6745e1 
10. Morrey, J.D., Wang, H., Hurst, B.L., Zukor, K., Siddharthan, V., Van Wettere, A.J., Sinex, D.G., and E.B. Tarbet (2018). Causation of Acute Flaccid Paralysis by Myelitis and Myositis in Enterovirus-D68 Infected Mice Deficient in Interferon $\alpha \beta / \gamma$ Receptor Deficient Mice. Viruses. 10(1), 33-55.

11. Nelson, G.R., Bonkowsky, J.L., Doll, E.; Green, M., Hedlund, G.L., Moore, K.R., and J.F. Bale, Jr. (2016). Recognition and Management of Acute Flaccid Myelitis in Children. Pediatr. Neurol. 55, pp. 17-21.

12. Pammolli, F., Magazzini, L., and M. Riccaboni (2011). The productivity crisis in pharamaceutical R\&D. Nat. Rev. Drug. Discov. 10(6), pp. 428-438.

13. Pastula, D.M., Aliabadi, N., Haynes, A.K., Messacar, K., Schreiner, T., Maloney, J., Dominguez, S.R., Davizon, E.S., Leshem, E., Fischer, M., et al. (2014). Acute neurologic illness of unknown etiology in children-Colorado, August-September 2014. Morb. Mortal. Wkly. Rep., 63, 901-902.

14. Platania, C.B.M., Leggio, G.M., Drago, F., Salomone, S., and C. Bucolo (2018). Computational systems biology approach to identify novel pharmacological targets for diabetic retinopathy. Biochem. Pharmacol. 158, pp. 13-26.

15. Sadiku, M.N.O., Tembely, M., and S.M. Musa (July 2018). Computational statistics, Inter. J. of Adv. Res. in Comp. Sci. and Software Eng., 8(7), pp. 76-78.

16. Sliwoski, G., Kothiwale, S., Meiler, J., and E.W. Lowe, Jr. (January 2014). Computational methods in drug discovery, Pharmacol. Rev, 66, pp. 334-395.

17. Vilar, S., Uriarte, E., Santana, L., Lorberbaum, T., Hripcsuk G., Friedman, C., and N.P. Tatonetti (2014). Similarity-based modeling in large-scale prediction of drug interactions. Nature Protocols, 9, pp. 2147-2163.

18. Weinhart M., Hocke, A., Hippenstiel, S., Kurreck, J., and S. Hedtrich (2018). 3D organ models - Revolution in pharmacological research?, Pharmacol. Res. Online: https://doi.org/10.1016/j.phrs.2018.11.002.

19. Wu, D.H. and X.J. Xu (2011). A New Practice: Study on the Molecular Mechanism of Traditional Chinese Medicine by Computational Pharmacology Methods: Part 1: Pharmacokinetic Modeling and Chemical Space Distribution, Letters in Drug Des. \& Discov., 8(7), pp. 652-658. 